\title{
Note on the Formation of Supermassive Black Holes
}

\author{
Kenneth Dalton \\ Th. Pongprasart, Bang Saphan, Prachuap Kiri Khan, Thailand \\ Email:kxdalton@yahoo.com
}

How to cite this paper: Dalton, K. (2020) Note on the Formation of Supermassive Black Holes. Journal of High Energy Physics, Gravitation and Cosmology, 6, 321-323. https://doi.org/10.4236/jhepgc.2020.63025

Received: May 1, 2020

Accepted: June 2, 2020

Published: June 5, 2020

Copyright $\odot 2020$ by author(s) and Scientific Research Publishing Inc. This work is licensed under the Creative Commons Attribution International License (CC BY 4.0). http://creativecommons.org/licenses/by/4.0/

\section{Abstract \\ Supermassive black holes formed during the lepton epoch of the Big Bang. What follows is a description of how this may have happened.}

Keywords

Supermassive Black Holes, Electron-Positron Model

\section{Introduction}

The electron-positron model of supermassive black holes is given in two recent papers [1] [2]. The equilibrium conditions from [1] are reproduced below. The intermediate masses (Table 1) range from $10^{3}$ to $8 \times 10^{6} M_{\odot}$. They are supported against gravity by electron degeneracy pressure and are characterized by the central Fermi energy $\epsilon_{F 0}$. They are in a quantum ground state and do not radiate. Larger masses (Table 2) are supported by ideal gas and radiation pressure, with a central thermal energy $k T_{0}$. They are equal in size to the Schwarzschild radius, so that the gas and radiation are confined.

Clusters of black holes can explain the dark matter in elliptical galaxies, dwarf galaxies, star clusters and galactic clusters [3]. Spiral galaxies evolved around a single black hole, and the missing mass must be treated separately [4]. In these

Table 1. Intermediate-mass.

\begin{tabular}{ccccc}
\hline$M\left(M_{\odot}\right)$ & $R>R_{s}(\mathrm{~cm})$ & $\rho_{0}\left(\mathrm{~g} \cdot \mathrm{cm}^{-3}\right)$ & $P_{0}(\mathrm{~Pa})$ & $\epsilon_{F 0}(\mathrm{eV})$ \\
\hline $10^{3}$ & $4.8\left(10^{13}\right)$ & $2.6\left(10^{-5}\right)$ & $3.9\left(10^{9}\right)$ & 2.1 \\
$10^{4}$ & $2.25\left(10^{13}\right)$ & $2.6\left(10^{-3}\right)$ & $8.4\left(10^{12}\right)$ & $4.5(10)$ \\
$10^{5}$ & $1.05\left(10^{13}\right)$ & $2.6\left(10^{-1}\right)$ & $1.8\left(10^{16}\right)$ & $9.6\left(10^{2}\right)$ \\
$10^{6}$ & $4.8\left(10^{12}\right)$ & $2.6(10)$ & $3.9\left(10^{19}\right)$ & $2.1\left(10^{4}\right)$ \\
$8\left(10^{6}\right)$ & $2.4\left(10^{12}\right)$ & $1.7\left(10^{3}\right)$ & $3.9\left(10^{22}\right)$ & $3.3\left(10^{5}\right)$ \\
\hline
\end{tabular}


Table 2. Supermassive.

\begin{tabular}{ccccc}
\hline$M\left(M_{\odot}\right)$ & $R=R_{s}(\mathrm{~cm})$ & $\rho_{0}\left(\mathrm{~g} \cdot \mathrm{cm}^{-3}\right)$ & $P_{0}(\mathrm{~Pa})$ & $k T_{0}(\mathrm{eV})$ \\
\hline $8\left(10^{6}\right)$ & $2.4\left(10^{12}\right)$ & $2\left(10^{3}\right)$ & $3.9\left(10^{22}\right)$ & $1.1\left(10^{5}\right)$ \\
$10^{7}$ & $3\left(10^{12}\right)$ & $1.3\left(10^{3}\right)$ & $2.5\left(10^{22}\right)$ & $1.1\left(10^{5}\right)$ \\
$10^{8}$ & $3\left(10^{13}\right)$ & $1.3(10)$ & $2.5\left(10^{20}\right)$ & $6.2\left(10^{4}\right)$ \\
$10^{9}$ & $3\left(10^{14}\right)$ & $1.3\left(10^{-1}\right)$ & $2.5\left(10^{18}\right)$ & $2.3\left(10^{4}\right)$ \\
$10^{10}$ & $3\left(10^{15}\right)$ & $1.3\left(10^{-3}\right)$ & $2.5\left(10^{16}\right)$ & $8\left(10^{3}\right)$ \\
$10^{11}$ & $3\left(10^{16}\right)$ & $1.3\left(10^{-5}\right)$ & $2.5\left(10^{14}\right)$ & $2\left(10^{3}\right)$ \\
\hline
\end{tabular}

and other cases, it is the arrival of black holes in the early Universe that helps to explain recent discoveries in astronomy.

\section{Formation of Black Holes}

A great profusion of electrons and positrons occurred during the lepton epoch of the Big Bang. It is estimated that for every nucleon there were $10^{9}$ leptons, so that the mass of leptons exceeded that of nucleons by a factor of $10^{6}$ [5]. It may be said that this period began at time $t=10^{-4} \mathrm{~s}$, temperature $T=10^{12} \mathrm{~K}$ and density $\rho=10^{14} \mathrm{~g} \cdot \mathrm{cm}^{-3}$, when the final creation of muons took place. This was followed by annihilation and by the decay of muons into electrons and neutrinos (but no photons). The latter reaction depleted the photon density in favor of electrons and positrons. The lepton energy density $u_{\text {lep }}=3 \rho_{\text {lep }} k T / m$ will be greater than that of radiation $u_{\text {rad }}=a T^{4}$ if

$$
\rho_{\text {lep }}>\frac{a m}{3 k} T^{3}
$$

The corresponding Jeans mass is [6]

$$
M_{J}=\left(\frac{750}{\pi}\right)^{1 / 2}\left(\frac{k}{G m}\right)^{3 / 2}\left(\frac{T^{3}}{\rho_{\text {lep }}}\right)^{1 / 2}<2 \times 10^{8} M_{\odot}
$$

Any mass greater than $M_{J}$ will be unstable toward gravitational collapse. In response to the surge in density, the lepton mass shattered into billions of spherical masses, each with a decreasing rate of expansion. They became supermassive black holes.

The time required for expansion from the highly compressed state to equilibrium may be estimated from $R / \mathrm{c}$ in the tables. This ranges from $10^{2}$ to $10^{6}$ seconds. During this length of time, a great deal of annihilation occurred. In the present-day Universe, the mass ratio of dark (leptonic) matter to normal (baryonic) matter is five or six to one. Comparison with the above-cited factor of $10^{6}$ shows that a minute portion of leptons achieved equilibrium as a black hole. The vast majority annihilated and replenished the expanding radiation field.

\section{Remark}

The conventional treatment of black holes is mathematical. It posits a solution to the field equations of general relativity, which yields a singular metric at the 
Schwarzschild radius [7]. This implies that light cannot escape from a black hole. However, this phenomenon was already known to Laplace and others [8]. Since that time, the difficulty has been to find a suitable concentration of mass. The electron-positron model solves that problem, and it provides a physical understanding of black holes.

\section{Conflicts of Interest}

The author declares no conflicts of interest regarding the publication of this paper.

\section{References}

[1] Dalton, K. (2019) Supermassive Black Holes. JHEPGC, 5, 984-988. https://doi.org/10.4236/jhepgc.2019.53052

[2] Dalton, K. (2014) The Galactic Black Hole. Hadronic J., 37, 241-245. http://vixra.org/abs/1404.0067

[3] Dalton, K. (2019) Black Hole Clusters: The Dark Matter. JHEPGC, 5, 989-991. https://doi.org/10.4236/jhepgc.2019.54053

[4] Dalton, K. (2013) The Missing Mass of the Milky Way Galaxy. Hadronic J., 36, 499. https://arxiv.org/abs/0905.3595

[5] Harrison, E. (2000) Cosmology. 2nd Edition, Cambridge University Press, Cambridge, Chap. 20. https://doi.org/10.1017/CBO9780511804540

[6] Carroll, B. and Ostlie, D. (2007) An Introduction to Modern Astrophysics. 2nd Edition, Addison-Wesley, San Francisco, Sect. 12.2.

[7] Adler, R., Bazin, M. and Schiffer, M. (1975) Introduction to General Relativity. 2nd Edition, McGraw-Hill, Tokyo, Sect. 6.8.

[8] Harrison, E. (2000) Cosmology. 2nd Edition, Cambridge University Press, Cambridge, Chap. 13. https://doi.org/10.1017/CBO9780511804540 